Sigerist, Henry E. Early mediaeval medical texts in manuscripts of Montpellier. (3 fig.) Bulletin of the History of Medicin 10, 27-52, (1941).

Stoll, A. Altes und Neues über Mutterkorn. Mitt. Naturf. Ges. Bern 1942, 45-80. P. Haupt Bern 1943.

Studer, German. Das Medizinalwesen im Wallis von 1798 bis 1930. Personen der Sanitätspolizei, Ärzte, Chirurgen, Geburtshelfer, Zahnärzte, Apotheker und Hebammen. Diss. med. Basel 1940.

Thorndike, Lynn. Three tracts on food in Basel manuscripts. Bull. Hist. of Med. 8, 355, (1940).

- Little known Medical works and authors in Basel manuscripts. Ann. of Med. Hist. 2, 280, (1940).

Tschirch, A(lexander). Das Mutterkorn und die Antönierbruderschaft. Schweiz. Apoth. Ztg. 77, 429-431, (1939).

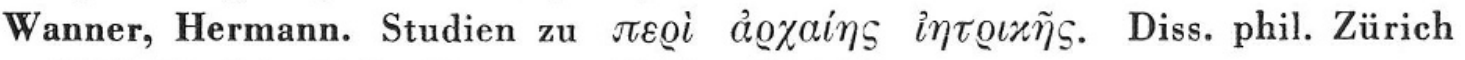
1939 (Buchdr. Gebr. Leemann \& Co., Zürich).

Wehrli, G. A. Die ärztliche Verordnung von Leibesübungen bei einigen Schweizer-Autoren des 18. Jahrhunderts. Festschrift Dr. Brodbeck-Sandreuter, Basel 1942, S. 21-72.

Wellauer, Wilhelm. Das Sanitätswesen im Niedersimmental in alter und neuer Zeit. Niedersimmental in Erlenbach 1937, $120 \mathrm{~S}$.

Wenger, Otto. Geschichte der Epilepsie. Ein Rückblick auf vier Jahrtausende. Monatsschrift f. Psychiatrie und Neurologie 106, 163, (1942).

Hans Fischer.

\title{
Zur Erinnerung an Antoine Laurent Lavoisier
}

(1743-1794)

Am 26. August 1743 in Paris geboren, in günstigen äußeren Verhältnissen heranwachsend, genoß Lavoisier eine sehr gründliche Erziehung, bei welcher hervorragende Lehrer mitwirkten. $\mathrm{Zu}$ diesen zählte der durch Begründung eines natürlichen Pflanzensystems hervorragende Bernard de Jussieu ${ }^{1}$ (1699-1777). Nach Absolvierung des Studiums der Rechte wandte sich der mathematisch Begabte aus innerer Neigung dem Studium der Naturwissenschaften, insbesondere der Chemie zu. Seine Stellung als Generalpächter (Fermier générale) gab ihm volle Unabhängigkeit. Die hohen Einkünfte verwandte er für seine bedeutende Mittel erfordernden Experimentalarbeiten. (Die Originalapparate Lavoisier's werden im «Conservatoire des Arts et Métiers» in Paris aufbewahrt.) Der Pariser Akademie gehörte er seit 1768 an. 1776 wurde er an die Spitze der Verwaltung der

1 Antoine Laurent de Jussieu (1748-1836) Professor am Jardin des Plantes zu Paris, der Neffe Bernards, setzte diese Arbeiten fort; er vertiefte vor allem die Erkenntnis der Verwandtschaftsverhältnisse der Pflanzen durch scharfe Hervorhebung der Familienmerkmale. 
staatlichen Salpeter- und Pulverfabrikation gestellt, 1790 in die Kommission zur Regulierung des Maß- und Gewichtssystems gewählt. Lavoisier hat seine außerordentlichen Geistesgaben jederzeit in den öffentlichen Dienst seines Vaterlandes gestellt, der Ruhm seiner wissenschaftlichen Leistung drang in alle Welt, die Chemie galt eine Zeit lang geradezu als französische Wissenschaft. Im Revolutionsjahr 1789 kam Lavoisier's epochemachender «Traité de Chimie» heraus. Die konstituierende Nationalversammlung nahm Lavoisier's Dienste weiter in Anspruch. Während der Schreckenszeit erinnerte man sich aber der einflußreichen Stellung, die er unter dem Königtum bekleidet hatte. Vor dem Revolutionstribunal, vor das ihn eine nichtige Beschuldigung gebracht hatte, halfen ihm seine hervorragenden Verdienste um das Wohl des Vaterlandes nichts. Als Lavoisier, zum Tode verurteilt, um 14tägigen Aufschub bat, um ein wichtiges Experiment zu Ende zu führen, bekam er vom Präsidenten des Tribunals, Coffinhal, die Antwort: «Nous n'avons plus besoin des savants».2 Das Gericht, welches dem blutigen Terror Robespierre's gehorchte, entschied auf Todesstrafe. Am 8. Mai 1794 fiel einer der besten Köpfe des jungen Frankreich der Guillotine zum Opfer. Vielleicht war Lavoisier das persönliche Opfer Marat's, den er sich zum Feind machte, weil er seine «Feuertheorie» ablehnend rezensiert hatte. ${ }^{3}$ Lavoisier starb gefaßt und ruhig den Tod der Revolution.

Seine Leistung liegt nicht so sehr auf dem Gebiet eigener Entdeckungen, als in der synthetischen Konzeption der Chemie, welche dank Lavoisier's Bemühungen eine einheitliche theoretische Auffassung auf quantitativer Grundlage ermöglichte. Damit wurde Lavoisier zum Begründer der modernen Chemie. Lavoisier's Denken war streng quantitativ; durch ihn wurde die Waage in den Dienst der Chemie grestellt. Auf seine Arbeiten gründet sich das Gesetz der Erhaltung der Materie. In dem «Traité élémentaire de Chimie» (1789), welches von seiner Frau in reizvoller Weise illustriert wurde, drückte er dieses Grundgesetz unmißverständlich aus: «Rien ne se crée ni dans les opérations de l'art, ni dans celles de la nature, et l'on peut poser en principe, que dans toute opération, il y a une égale quantité de matière avant et après l'opération»...

Lavoisiers Lebensarbeit galt dem Problem der Verbrennung, welches ihn bald (von

2 H. Taine, Les origines de la France contemporaine. La Révolution. Tom III, p. 459, Paris 1887.

3 Jean-Paul Marat, seit frühester Jugend von unersättlichem Ehrgeiz erfüllt und von grenzenloser Ruhmsucht getrieben, unfähig, die großen Leistungen seiner Zeitgenossen Laplace, Lavoisier, Fourcroy auch nur zu verstehen, ohne eigene Originalität, vom Drang zum «Gelehrten» besessen, veröffentlichte er seine «Entdeckungen». So schrieb er z. B. «Mémoires académiques sur la lumière», in welchen er Newton bekämpfte. Oder er verfaßte ein «Traité de l'homme», welches «zum ersten Mal» die Beziehungen zwischen Leib und Seele «klarlegte». In der Art megalomaner Psychopathen sagt er über seine (ganz wertlose) «Feuertheorie»: «Enfin, le précis de mes expériences sur le feu vit le jour: la sensation qu'il fit en Europe fut prodigieuse; tous les papiers publics en firent mention; pendant sit mais, j'eus chez moi la cour et la ville ... L'Académie, ayant reconnu qu'elle ne pouvait pas étouffer mes découvertes, chercha a les faire naître dans son sein.» ...

«C'est le délire ambitieux, bien connu dans les asiles» schreibt Taine (Hist. de la France contemporaine. La Révolution, Tome III, p. 165 [1887].) Ein Mann, der so von sich dachte, konnte keinen Widerspruch des wirklich «Gelehrten» ertragen. So tritt uns der Tod Lavoisiers auf der Guillotine gleichzeitig als Rache des Unbegabten am Genie entgegen.

${ }^{4}$ Frau Lavoisier lebte bis 1836. Sie publizierte 1806 einige Erinnerungen an ihren 
1777 an) mit den Vertretern der von Georg Ernst Stahl (1660-1743) begründeten Phlogistonlehre 5 in offenen Konflikt brachte.

Die Entdeckung des Sauerstoffs (1774) durch Josef Pristley (1733-1804) gab Lavoisier die Lösung zum Verständnis der Verbrennungsvorgänge in die Hand. Lavoisier verdanken wir die bis heute geläufige Auffassung von Oxydation und Reduktion, die durch ihn zur streng quantitativen Theorie ausgestaltet wurde. Er erkannte Kohlensäure und Wasser als Verbrennungsprodukte jeder organischen Substanz. - Henry Cavendish's (1731-1810) Entdeckung, daß sich bei Vereinigung von Wasserstoff mit Sauerstoff ausschließlich Wasser bildet (1781), gab ihm gleichzeitig die Erklärung für den früher nie richtig gedeuteten Prozeß der Auflösung eines Metalles in Säuren.

In polemischer Art behandelte Lavoisier «antiphlogistische» Fragen in dem «Mémoire sur la Combustion en générale» (1777), viel schärfer in den «Réflexion sur le Phlogistique» (1783). Auf Grund ausgedehnter quantitativer Verbrennungsversuche gelangte er zu der Vorstellung, daß Kohlenstoff, Wasserstoff und Sauerstoff die einzigen Bestandteile organischer Verbindungen seien. Damit war der unabsehbare Weg zur organischchemischen Analyse eröffnet.

Für die tierische Physiologie sind die Anschauungen Lavoisier's von grundlegender Bedeutung geworden, seine Arbeiten über den «Atmungsprozeß 17776 und 17897 zusammen mit dem Physiologen Armand Séguin (1765-1835) lieferten den Beweis, daß die Atmung einen Verbrennungsprozeß unter Bildung von Kohlensäure darstellt, welcher dem tierischen Organismus die Energie zur Erzeugung der tierischen Wärme liefert. 8

Zum exakten (quantitativen) Beweis dieser Auffassung konnte Lavoisier erst gelangen, nachdem Laplace (1749-1827) die moderne Grundlage der Wärmelehre geliefert und damit genaue calorimetrische Messungen ermöglicht hatte (vergl. A. L. Lavoisier et P.S. de Laplace, Mémoire sur la chaleur. Mém. de l'Acad. des Sciences, Paris 1780.) So datiert seit Lavoisier eine neue Epoche in der Erkenntnis der Oxydations- und Verbrennungsprozesse in der anorganischen, organischen und biologischen Welt. Sie stellt eine Erkenntnis so fundamentaler Art dar, daß wir dieses scharfsinnigen chemischen Denkers und großzügigen Experimentators in seinem 200. Geburtsjahr mit Dankbarkeit gedenken.

Hans Fischer.

5 Dargestellt in: Georg Ernst Stahl, Zymotechnica fundamentalis sive fermentationis theoria generalis ..., Halle 1697.

6 A. L. Lavoisier, Expériences sur la respiration des animaux ... Mémoire de l'Acad. des Sciences, Paris 185 (1777).

7 A.L.Lavoisier et A.Séguin, Sur la respiration des animaux. Mém. de l'Académie des Sciences, Paris 1789, p. 185.

8 Als Vorläufer von Lavoisier's Theorie der tierischen Verbrennung ist der genau 100 Jahre vor Lavoisier geborene jung verstorbene Engländer John Mayow (1643-1679) nicht zu vergessen, welcher das Wesen der Verbrennungs- und Atmungschemie im modernen Sinn auf Grund genial angelegter Experimente bereits richtig zu erkennen und deuten vermochte. Ist doch der «spiritus nitroaereus» Mayow's nichts anderes als der 100 Jahre später von Lavoisier so benannte Sauerstoff. Doch blieb Mayow's Auffassung, welche für die Entwicklung der Physiologie ebenso epochemachend hätte werden können wie Lavoisier's Entdeckung, völlig vergessen. 